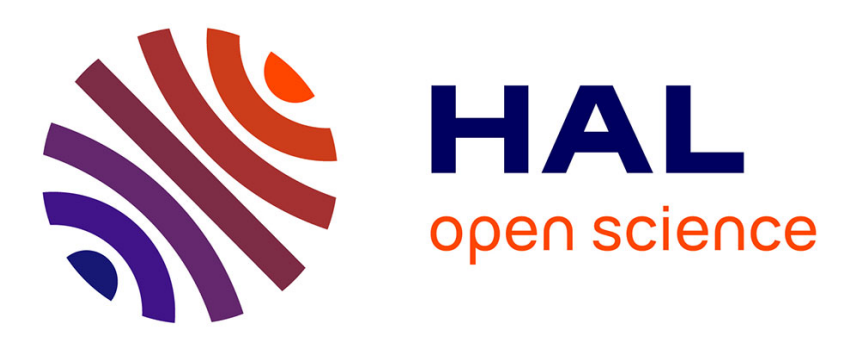

\title{
A new model for the phase transitions due to octahedra rotations in the perovskite structure \\ M Rousseau
}

\section{To cite this version:}

M Rousseau. A new model for the phase transitions due to octahedra rotations in the perovskite structure. Journal de Physique Lettres, 1979, 40 (17), pp.439-443. 10.1051/jphyslet:019790040017043900 . jpa-00231661

\section{HAL Id: jpa-00231661 https://hal.science/jpa-00231661}

Submitted on 1 Jan 1979

HAL is a multi-disciplinary open access archive for the deposit and dissemination of scientific research documents, whether they are published or not. The documents may come from teaching and research institutions in France or abroad, or from public or private research centers.
L'archive ouverte pluridisciplinaire HAL, est destinée au dépôt et à la diffusion de documents scientifiques de niveau recherche, publiés ou non, émanant des établissements d'enseignement et de recherche français ou étrangers, des laboratoires publics ou privés. 


\title{
A new model for the phase transitions due to octahedra rotations in the perovskite structure
}

\author{
M. Rousseau \\ Laboratoire de Physique de l'Etat Condensé (*), Faculté des Sciences, 72017 Le Mans Cedex, France \\ (Reçu le 26 décembre 1978, révisé le 22 juin 1979, accepté le 4 juillet 1979)
}

\begin{abstract}
Résumé. - Nous proposons un modèle ordre-désordre décrivant les transitions de phase dues aux rotations des octaèdres $\mathrm{MX}_{6}$ dans la structure pérovskite. Les distorsions cristallines et l'apparition d'un pic central sont discutées en considérant les défauts intrinsèques dus au désordre associé à la rotation des octaèdres rigides $\mathbf{M X}_{6}$.

Abstract. - We give an order-disorder picture of the phase transitions due to $\mathrm{MX}_{6}$ octahedra rotations in the perovskite structure. The lattice distortions and the occurrence of a central peak are discussed in terms of the intrinsic defects resulting from the disorder associated with the rotation of $\mathbf{M X}_{6}$ rigid octahedra.
\end{abstract}

1. Introduction. - Many cubic perovskite-type crystals of general formula $\mathrm{AMX}_{3}$ undergo phase transitions due to the tilting of $\mathrm{MX}_{6}$ octahedra around $\langle 100\rangle$ axes. It has been admitted that these broken symmetries are caused by the condensation of the particular normal mode of the phonon in the high temperature phase whose eigenvectors correspond to the static atomic displacement in the structure stabilized below the transition temperature $T_{\mathrm{c}}$. In fact the theoretical models trying to explain the occurrence of a central peak in addition to the soft phonon imply that even for such displacive systems the time averaged structure of the high temperature phase is of a disordered nature. Many double well potential models have been extensively developed recently [1] and the aim of this paper is to concretely suggest (without any theoretical elaboration) a feasible multiple well potential for the $\mathrm{X}$ atoms of the $\mathrm{MX}_{6}$ octahedra in the perovskite structure and relate this point of view to the crystallographic results.

2. Description of the model. - The basis concepts of this model lie essentially with the two following features :

- In the perovskite structure, phase transitions due to octahedra rotations occur when the spacing between the $\mathrm{M}$ and $\mathrm{X}$ ions (Fig. 1) is smaller than the sum of their ionic radii [2]. So the $\mathrm{X}$ ions are

$\left.{ }^{*}\right)$ E.R.A. $n^{\circ} 682$ pushed out from their ideal positions in the plane perpendicular to the M-X-M direction.

- As previously mentioned in ref. [3], the phonon dispersion curves of these compounds show that the modes which leave the octahedra undistorted are much smaller than the others. So when looking at the phenomena related to these low frequency modes (octahedra rotations) the $\mathbf{M X}_{6}$ octahedra can be considered as rigid.

We then concluded that in the perovskite compounds where $\mathrm{MX}_{6}$ octahedra rotations are expected, a disorder associated with the tilting of undeformable $\mathrm{MX}_{6}$ octahedra is feasible and we assumed that the potential energy of each $X$ atom is represented by a Mexican hat (Fig. 1) with four minima on the brim (in accordance with the symmetry of the sites occupied by these atoms) [4]. So, each $\mathrm{MX}_{6}$ octahedron has the choice between 8 possible orientations noted $\left(\alpha^{ \pm}, \beta^{ \pm}, \gamma^{ \pm}\right)$when $\alpha, \beta$ and $\gamma$ represent the tilting angles around the $O x, O y$ and $\mathrm{Oz}$ directions respectively.

In the high temperature phase, the $\mathrm{X}$ atoms can easily go over the top of the hat leading to a completely disordered cubic phase (Fig. 2). The mean atomic position is then the ideal perovskite structure with a large Debye-Waller factor for the $X$ atoms $[5,6]$. When the temperature is lowered, two limit cases may be envisaged according to the relative values of $\Delta_{1}$ and $\Delta_{2}-\Delta_{1}$.

i) $\Delta_{2}-\Delta_{1} \ll \Delta_{1}$

The disordered cubic phase will lead to a rhom- 


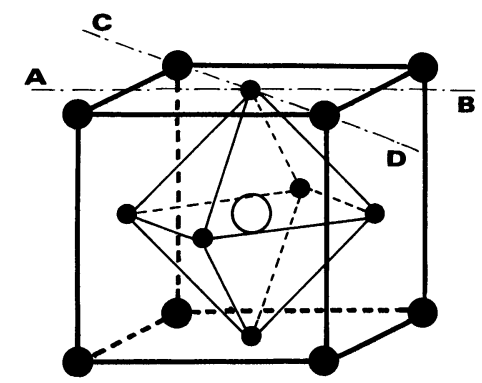

$\bullet \mathrm{A}^{+} \quad \mathrm{OM}^{++} \bullet \mathrm{X}^{-}$
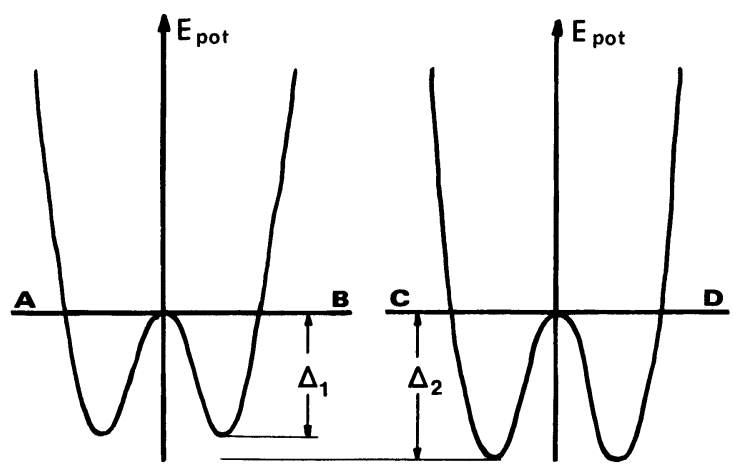

Fig. 1. - Shape of the single particle potential for the $\mathrm{X}$ atoms of the perovskite structure. The instantaneous position of a particle lies in one of the four minima along the $\langle 110\rangle$ directions. $\left(\Delta_{2}-\Delta_{1} \ll \Delta_{1}\right.$ leads to the dynamical picture described in figure 4 , whereas $\Delta_{2}-\Delta_{1} \sim \Delta_{1}$ may induce a static rotation around the three fourfold axes leading to a rhombohedral phase as observed in LaAlO ${ }_{3}$ ).

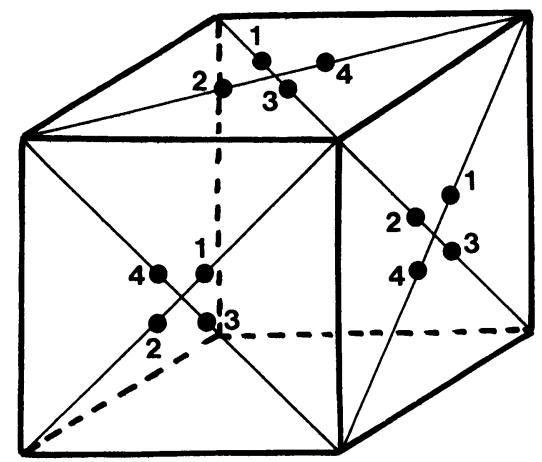

Fig. 2. - Expected high temperature disordered perovskite structure.

bohedral low temperature phase (such as the one observed in $\mathrm{LaAlO}_{3}$ [7]) in which the octahedra remain tilted about each of the $\langle 100\rangle$ axes.

ii) $\underline{\Delta}-\Delta_{1} \gg \Delta_{1}$

When the temperature is low enough to prevent $\mathrm{X}$ atoms from going over the top of the hat, the atoms can however have an angular motion in the bottom of the brim. Under these conditions, when an $X$ atom moves about its four potential minima, the motion of the corresponding undeformable $\mathbf{M X}_{6}$ octahedra is described either by one or the other of the two configurations $\Gamma^{+}$and $\Gamma^{-}$represented in figure 3. The $\Gamma^{+}$configuration corresponds to the succession :

$$
\begin{aligned}
1\left(\alpha^{+}, \beta^{-}, \gamma^{+}\right) \rightarrow 2\left(\alpha^{+}, \beta^{+}, \gamma^{+}\right) \rightarrow \\
\\
\rightarrow 3\left(\alpha^{-}, \beta^{+}, \gamma^{+}\right) \rightarrow 4\left(\alpha^{-}, \beta^{-}, \gamma^{+}\right)
\end{aligned}
$$

whereas the $\Gamma^{-}$configuration is obtained by taking $\gamma^{-}$instead of $\gamma^{+}$. As shown on figure 4, this dynamical disorder $\left(\alpha^{ \pm}, \beta^{ \pm}\right)$induces a static rotation of the octahedra around the $z$ axis $\left(\gamma^{+}\right.$or $\left.\gamma^{-}\right)$. Furthermore, each $\mathrm{X}$ atom being shared by two octahedra, the static rotations are strongly correlated in the plane perpendicular to the $z$ axis and not along this axis. Then for a cubic to tetragonal phase transition occurring at $T_{\mathrm{c}}$, a $z$ axis monodomain of the quadratic
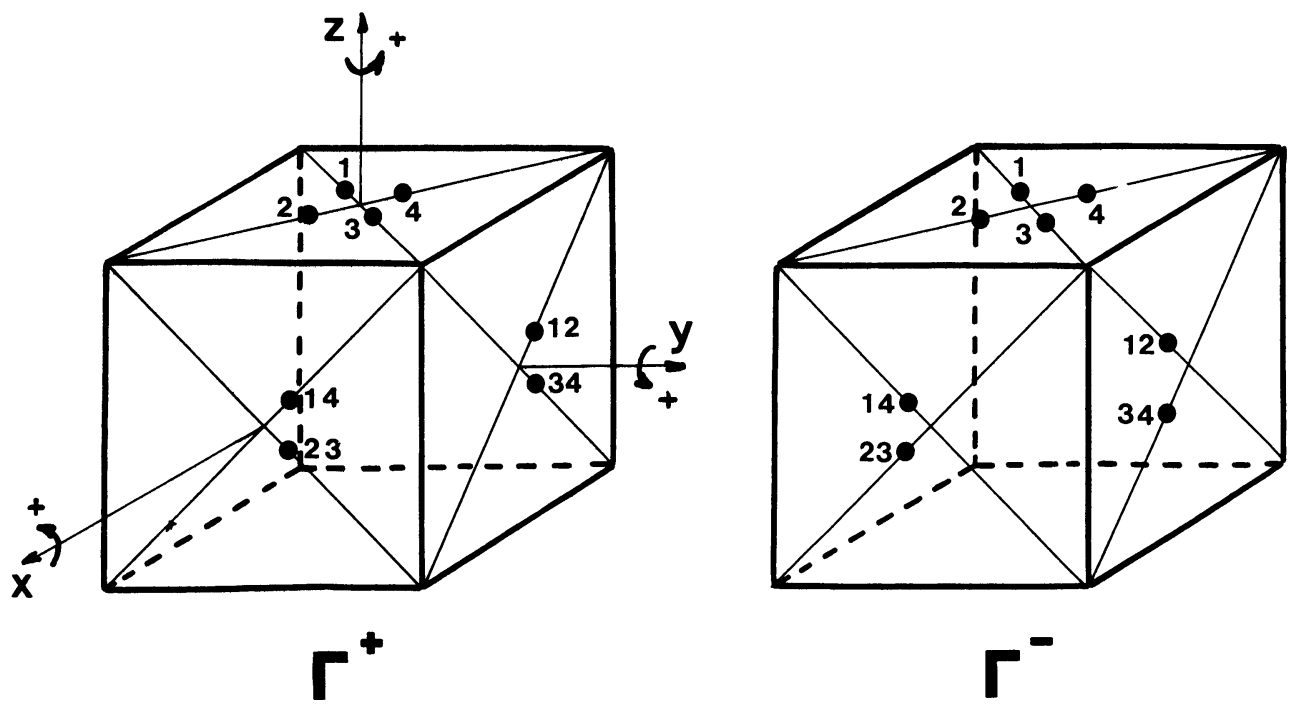

Fig. 3. - The two $\Gamma^{+}$and $\Gamma^{-}$configurations resulting from the same motion of an $\mathrm{X}$ atom of a rigid $\mathrm{MX}_{6}$ octahedron between its four equilibrium positions. 


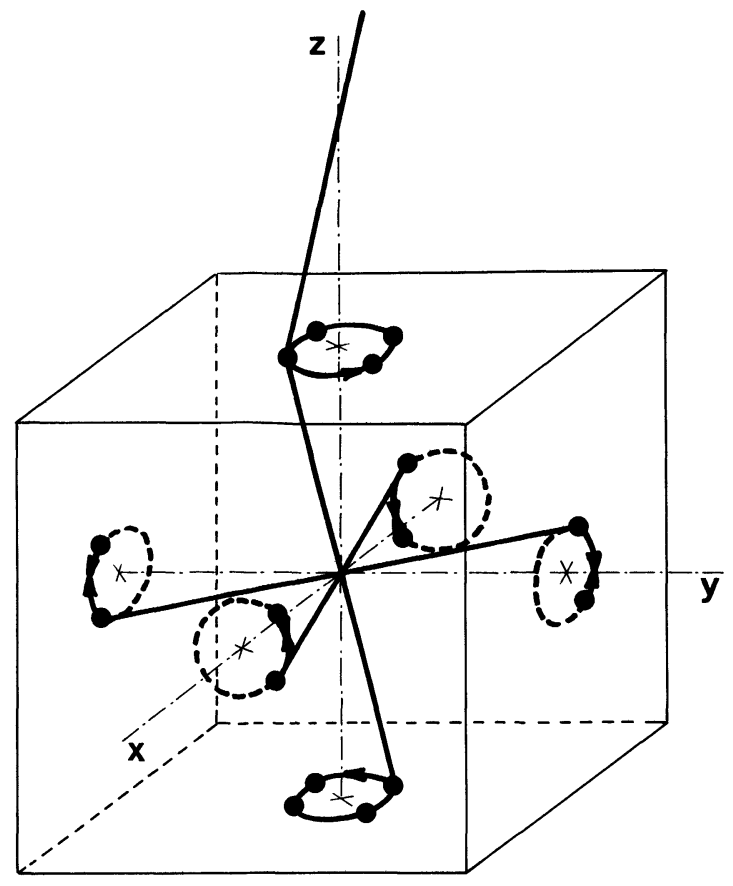

Fig. 4. - Precessing motion of a $\mathbf{M X}_{6}$ rigid octahedron in a $z$ axis monodomain. In the cubic phase $\alpha=\beta=\gamma=\varphi_{0}$; in the quadratic phase $\alpha=\beta \neq \gamma$.

phase is a perfectly ordered pile of the correlated planes described above. The well-known distorted perovskite structures of symmetry $\mathrm{D}_{4 \mathrm{~h}}^{5}$ and $\mathrm{D}_{4 \mathrm{~h}}^{18}$ correspond respectively to the mean atomic positions of a sequence $\Gamma^{+}-\Gamma^{+}-\Gamma^{+} \ldots$ or $\Gamma^{+}-\Gamma^{-}-\Gamma^{+}-\Gamma^{-} \ldots$ along the $z$ axis. These two phases are associated respectively to superlattice Bragg reflections at the $(h+1 / 2, k+1 / 2, l) \mathrm{M}$ points or the $(h+1 / 2$, $k+1 / 2, l+1 / 2) \mathrm{R}$ point, of the cubic Brillouin zone.

This model can give a qualitative interpretation of the orientating mechanism leading to the anisotropy of the local fluctuations observed in $\mathrm{SrTiO}_{3}$ by Von Waldkirch [8] from E.P.R. experiments and by E. Courtens [9] from birefringence data. In fact, in the vicinity of $T_{\mathrm{c}}$, in the high temperature phase, we can introduce an intermediate region in which the embryo of a $z$ axis domain is a disordered pile of correlated planes (e.g. $\Gamma^{+}-\Gamma^{+}-\Gamma^{+}-\Gamma^{+}-\Gamma^{-}-\Gamma^{-} \ldots$ ) without any instantaneous periodicity along the $z$ axis. Then the bidimensional correlations induce the diffuse scattering observed by Comes et al. [10] in $\mathrm{KMnF}_{3}$ and $\mathrm{NaNbO}_{3}$ all along the R-M lines parallel to the $q_{z}$ direction of a $z$ axis cubic domain.

3. Lattice distortion. - In the conventional displacive description of these phase transitions the decreasing of the two equal lattice parameters $\left(a_{\mathrm{q}}=b_{\mathrm{q}}\right)$ in the quadratic phase is directly related to the angle of tilt $\gamma$ of the octahedra about the $z$ axis by [11] :

$$
a_{\mathrm{q}}=b_{\mathrm{q}}=a_{\mathrm{c}} \cos \gamma
$$

where $a_{\mathrm{c}}$ is the pseudo-cubic lattice parameter $\left(a_{\mathrm{c}}=\sqrt[3]{a_{\mathrm{q}}^{2} c_{\mathrm{q}}}\right)$

On the other hand, this theory cannot explain the increase of $c_{\mathrm{q}}$ in the quadratic phase $\left(c_{\mathrm{q}}>a_{\mathrm{c}}\right.$ [2]) without the introduction of a phenomenological coupling between strain and order parameter [12] inducing a quadratic deformation of the octahedra. In absence of any appreciable experimental change in the thermal expansion coefficient at the transition $\left(c_{\mathrm{q}}-a_{\mathrm{c}}=2\left|a_{\mathrm{c}}-a_{\mathrm{q}}\right|\right)$ the tilt angle $\gamma$ can be related approximately to the lattice parameters $a_{\mathrm{q}}$ and $b_{\mathrm{q}}$ by

$$
\frac{c_{\mathrm{q}}-a_{\mathrm{q}}}{a_{\mathrm{q}}} \simeq \frac{3}{2} \gamma^{2} .
$$

In fact this point of view is not consistent with the observation of regular or slightly distorted octahedra in the low symmetry phases of $\mathrm{NaNbO}_{3}$ [13], $\mathrm{SrZrO}_{3}$ [14], $\mathrm{NaNiF}_{3}$ [15], $\mathrm{KCaF}_{3}$ [16] or $\mathrm{RbCaF}_{3}$ [17].

Let us see now how the model described below can account for the observed lattice distortion. In a $z$ axis monodomain of the cubic phase, the tilting angles $\alpha, \beta, \gamma$, around the $|100|,|010|$ and $|001|$ directions respectively are equal to $\varphi_{0}$ (Fig. 4). If $a(T)$ is the $\mathrm{M}-\mathrm{X}$ bond length, each $\mathrm{X}$ atom being concerned by two octahedra rotations around two of the 3 fourfold axes, the lattice parameter $a_{\mathrm{c}}$ in the cubic phase is given by :

$$
a_{\mathrm{c}}=a(T) \cos ^{2} \varphi_{0}
$$

In the quadratic phase, the decrease of $a_{\mathrm{q}}=b_{\mathrm{q}}$ is due to the increase of $\gamma$ whereas the evolution of $c_{\mathrm{q}}$ is attributed to the decrease of $\alpha$ and $\beta$.

Thus :

$$
\begin{gathered}
a_{\mathrm{q}}=b_{\mathrm{q}}=a(T) \cos \left(\varphi_{0}+\mathrm{d} \gamma\right) \cos \left(\varphi_{0}+\mathrm{d} \alpha\right) \frac{\mathrm{d} \gamma}{\mathrm{d} T}>0 \\
c_{\mathrm{q}}=a(T) \cos ^{2}\left(\varphi_{0}+\mathrm{d} \alpha\right) \quad \frac{\mathrm{d} \alpha}{\mathrm{d} T}=\frac{\mathrm{d} \beta}{\mathrm{d} T}<0 .
\end{gathered}
$$

To get an order of magnitude of $\varphi_{0}, d \alpha$ and $d \gamma$, we consider that in the absence of lower temperature phase transition, the quadratic structure is entirely ordered at $0 \mathrm{~K}$.

So

$$
c_{\mathrm{q}}(0)=a(0) \quad\left(\mathrm{d} \alpha=-\varphi_{0}\right) .
$$

Then, assuming that $a(T)$ follows the same law as the pseudo-cubic lattice parameter $\sqrt[3]{a_{\mathrm{q}}^{2} c_{\mathrm{q}}}$ (Fig. 5), we can easily evaluate $\varphi_{0}, \mathrm{~d} \alpha(T), \mathrm{d} \gamma(T)$ from the lattice parameters $a_{\mathrm{q}}$ and $c_{\mathrm{q}}$ deduced from X-ray measurements in $\mathrm{SrTiO}_{3}$ [18], $\mathrm{RbCdF}_{3}$ [2] and $\mathrm{RbCaF}_{3}$ [19]. The calculated values reported in figure 6 indicate that $\mathrm{d} \gamma(T)$ follows a Landau type behaviour of the form $A\left(T_{\mathrm{c}}-T\right)^{1 / 2}$; the lack of volume discontinuity at the transition implies that $\mathrm{d} \alpha+\mathrm{d} \beta+\mathrm{d} \gamma=0$ near $T_{\mathrm{c}}$. We may also notice that $\varphi_{0}$ is much smaller for $\mathrm{SrTiO}_{3}$ than for the fluoperovskites, the corresponding displacements of the 


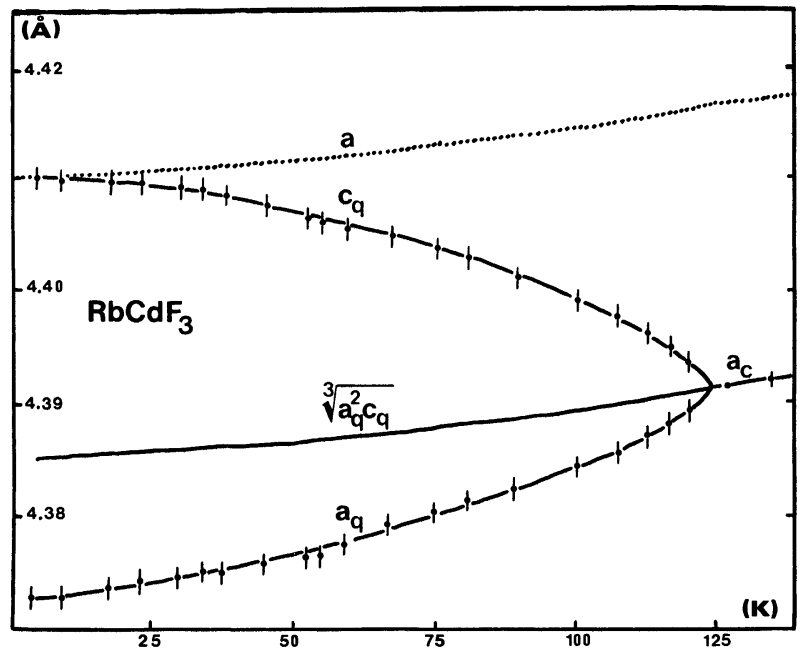

Fig. 5. - Quadratic and pseudo-cubic lattice parameters $v s$. temperature for $\mathrm{RbCdF}_{3}$ [2].
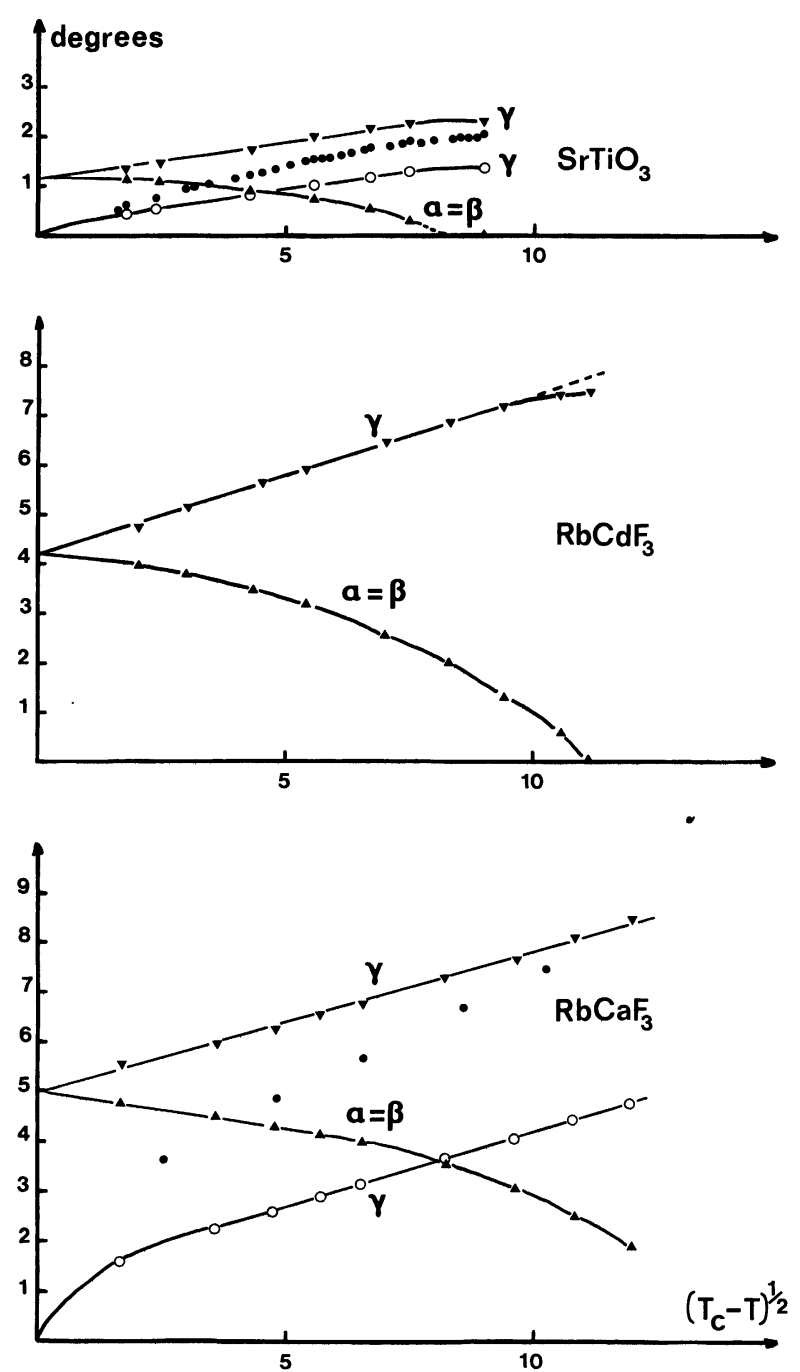

Fig. 6. - Temperature dependence of $\alpha=\beta(\Delta)$ and $\gamma(\nabla)$ according to the disordered model. (The black points correspond to the experimental values deduced from E.P.R. measurements in $\mathrm{SrTiO}_{3}$ [24] and neutron diffraction in $\mathrm{RbCaF}_{3}$ [17] ; the open circles represent the tilt angle $\gamma$ deduced from X-ray measurements in the classical displacive description).
$\mathrm{X}$ atoms in the cubic phase near $T_{\mathrm{c}}$ are $0.04 \AA$ for $\mathrm{SrTiO}_{3}$ and $0.2 \AA$ for $\mathrm{RbCaF}_{3}\left(0.5 \AA\right.$ for $\mathrm{CsPbCl}_{3}$ after Möller [20]). It appears that the oxygen probability distribution function in $\mathrm{SrTiO}_{3}$ is less anharmonic than that of fluorine in fluoperovskites in agreement with recent high resolution studies of anharmonic motion performed in $\mathrm{SrTiO}_{3}$ and $\mathrm{KMnF}_{3}$ [21] and with the underdamped character of the soft phonon in $\mathrm{SrTiO}_{3}$ [22].

Figure 6 shows that in $\mathrm{SrTiO}_{3}$ and $\mathrm{RbCaF}_{3}$, far from $T_{\mathrm{c}}$, the measured tilt angle is closer to the value predicted by the disordered model than to that given by the classical displacive model. The agreement is better with the neutron scattering data in $\mathrm{RbCaF}_{3}$ [23], [17] than with the E.P.R. data in $\mathrm{SrTiO}_{3}$ [24] but we may point out that if an order-disorder process plays an effective role in the mechanism of the transition, the tilt angle measured with E.P.R. on an impurity site can be different from the true tilt angle in the bulk crystal. On the other hand, the large discrepancy between our model and the experimental points in the vicinity of $T_{\mathrm{c}}$ will be discussed elsewhere [17] in terms of the thermal vibrations associated with the soft mode.

4. Discussion. - The model described above introduces three distinguishable domains (precursorclusters) in the cubic phase near $T_{\mathrm{c}}$. In a $z$ axis cluster (embryo of a $z$ axis quadratic monodomain) the effective order parameter dimensionality is $n=1$ (ordering along the $z$ axis) and a central peak is expected when the average value of the ordering variable is non zero on a timescale which is long compared with the inverse phonon frequencies. Furthermore, we can argue that any extrinsic impurity favouring the formation of a $z$ axis monodomain in the cubic phase will strongly influence this central peak. For example, any defect trapping such a domain (by anchoring its wall) or any anisotropic strain will increase its lifetime and lead to a lowering of the energy width of this peak. In a few words, we expect that a perovskite compound free from any strain and extrinsic impurity may exhibit a rather broad central peak near $T_{\mathrm{c}}$ but the energy width of this central peak may decrease when increasing the concentration of any defects favouring the quadratic symmetry.

Until this point of view is confirmed experimentally it will remain a simple idea which can nevertheless conciliate the intrinsic and the extrinsic theories trying to account for the occurrence of a central peak in perovskite compounds. In fact, the single potential described in this paper is no more than a peculiar concrete shape of the general double-well potential model commonly used in the intrinsic theories [1]. On the other hand, the flipping of the octahedra between the equivalent positions (symmetry restoring mode [25]), which is a linear combination of octahedra 
rotations around the fourfold axes, is necessary linearly coupled to the order parameter as the intrinsic defects considered by Halperin and Varma [26].

Although high resolution neutron scattering structural investigations are not yet able to derive a multiple peak probability distribution function on the oxygens of $\mathrm{SrTiO}_{3}$, the fluorines of $\mathrm{KMnF}_{3}$ [6] and the chlorines of $\mathrm{CsPbCl}_{3}$ [27], we may notice that a recent E.P.R. experiment clearly indicates that an orderdisorder local behaviour occurs in $\mathrm{SrTiO}_{3}$ near $T_{\mathrm{c}}$ [28] ; in the same way, ${ }^{87} \mathrm{Rb} \mathrm{NMR}$ study in $\mathrm{RbCaF}_{3}$ [29] seems to indicate a local symmetry lowering far above $T_{\mathrm{c}}$. In this context, $\mathrm{RbCaF}_{3}$ with its large tilt angles is one of the most suitable compound for testing the validity of this description ; to this purpose NMR spin-lattice relaxation time measurements are being carried out.

Acknowledgments. - This work is a part of my thesis, I am indebted to J. Nouet and A. Zarembowitch who initiated it as well as to M. Lambert, B. Dorner, J. Joffrin and K. A. Muller who accepted to be in the examining board. I am very grateful to the referees for their critical remarks.

\section{References}

[1] Krumhansl, J. A. and Schrieffer, J. R., Phys. Rev. B 11 (1975) 3535 ;

Aubry, S., J. Chem. Phys. 64 (1976) 3392;

Bruce, A. D. and Schneider, T., Phys. Rev. B 16 (1977) 3991.

[2] Rousseau, M., Gesland, J. Y., Julliard, J., Nouet, J., Zarembowitch, J. and Zarembowitch, A., Phys. Rev. B 12 (1975) 1579.

[3] Rousseau, M., Nouet, J. and Almairac, R., J. Physique 38 (1977) 1423.

[4] Rousseau, M., Thesis, Le Mans (1977).

[5] Harada, J., Sakata, M., Hoshino, S. and Hirotsu, S., $J$. Phys. Soc. Japan 40 (1976) 212.

[6] Meyer, G. M., Nelmes, R. J. and Hutton, J., in Proceedings of the International Conference on Lattice Dynamics, Paris, 1977.

[7] Kuems, J. K., Shirane, G., Muller, K. A. and Scheel, H. J., Phys. Rev. B 8 (1973) 1119.

[8] Von Waldkirch, Th., Muller, K. A. and Berlinger, W., Phys. Rev. B 7 (1973) 1052.

[9] Courtens, E., Phys. Rev. Lett. 29 (1972) 1380.

[10] Comes, R., Denoyer, F. et Lambert, M., J. Physique Colloq. 32 (1971) C5-195;

COMEs, R., Thesis, Orsay (1969); Denoyer, F., Thesis, Orsay (1977) and references therein.

[11] Alefeld, B., Z. Phys. 222 (1969) 155.

[12] Slonczeski, J. C. and Thomas, H., Phys. Rev. B 1 (1970) 3599.

[13] Ahtee, M., Glazer, A. M. and Megaw, H. D., Philos. Mag. 26 (1972) 995.
[14] Ahtee, A., Ahtee, M., Glazer, A. M. and Hewat, A. W., Acta Crystallogr. B 32 (1976) 3243.

[15] Hidaka, M. and Ono, M., J. Phys. Soc. Japan 43 (1977) 258.

[16] Hewat, A. W., Bulou, A. and Nouet, J., to be published.

[17] Bulou, A., Rousseau, M., Ridou, C., Nouet, J. and Hewat, A. W., to be published.

[18] Okazaki, A. and Kawaminami, M., Mat. Res. Bull. 8 (1973) 545.

[19] Ridou, C., Rousseau, M., Gesland, J. Y., Nouet, J. and ZAREMBowitch, A., Ferroelectrics 12 (1976) 199

[20] MфLLER, C. K., Mat. Fys. Medd Danske Vidensk. Selsk. 32 (1959) $\mathrm{n}^{\circ} 2$

[21] Nelmes, R. J., Private communication.

[22] Shapiro, S. M., Axe, J. D., Shirane, G. and Riste, T., Phys. Rev. B 6 (1972) 4332.

[23] Maetz, J., Müllner, M. and JeX, H., Phys. Status Solidi (a) 50 (1978) K 117.

[24] Muller, K. A., Berlinger, W. and Waldngr, F., Phys. Rev. Lett. 21 (1968) 814.

[25] Shigenari, T., Phys. Status Solidi (b) 64 (1974) 659.

[26] Halperin, B. I. and Varma, C. M., Phys. Rev. B 14 (1976) 4030.

[27] Hutton, J., Nelmes, R. J. and MEYeR, G. M., to be published,

[28] Bruce, A. D., Muller, K. A. and Berlinger, W., Phys. Rev. Lett. 42 (1979) 185.

[29] Bulou, A., Theveneau, H., Trokiner, A. and Papon, P., J. Phys. Lett., to be published. 